\title{
Artroplastia total de rodilla no cementada
}

\author{
Total noncemented knee arthroplasty
}

\section{Darío E Garín Zertuche, * José Iván Hernández Moriel, ${ }^{\ddagger}$ José Alfredo Penagos Paniagua§}

\begin{abstract}
* Cirujano Ortopedista. Egresado Instituto Nacional de Rehabilitación. Diplomado Artroscopia y Cirugía Articular. Subespecialidad en Reconstrucción Articular. Médico adscrito Hospital Ángeles. Tijuana, Baja California.

¥ Médico Residente de cuarto año de la Especialidad en Ortopedia y Traumatología. Hospital Christus Muguerza del Parque. Chihuahua, Chihuahua.

$\S$ Cirujano Ortopedista y Traumatólogo, Maestro en Ciencias Médicas. Adscrito a la Clínica de Artroscopia y Reemplazo Articular de Rodilla, Hospital Regional de Especialidades Núm. 30 del IMSS, Mexicali, Baja California.
\end{abstract}

Correspondencia: Darío E Garín Zertuche. Hospital Ángeles Tijuana, Paseo de los Héroes No. 10999-301, Zona Río 22010, Tijuana, Baja California. Correo electrónico: dgarinmd@gmail.com

\section{RESUMEN}

El interés por la artroplastia total de rodilla no cementada (ATR) ha aumentado considerablemente en los últimos años. A medida que el número de pacientes más jóvenes y más activos tratados con ATR continúa incrementando, se requiere considerar una mejor fijación como medio para optimizar la longevidad del implante. Esta técnica, menos utilizada que el reemplazo de rodilla cementado en las últimas décadas, revive gracias a un avance en el diseño protésico, la instrumentación y la técnica quirúrgica. La literatura relacionada muestra en algunos casos datos contradictorios sobre la supervivencia y la tasa de revisión, pero en la mayoría de los casos se describe una tasa de éxito comparable con la ATR cementada. Los componentes no cementados ofrecen la ventaja potencial de una interfaz biológica entre el hueso y los implantes, lo que podría demostrar mayor ventaja en la fijación duradera a largo plazo en el seguimiento de pacientes jóvenes sometidos a ATR. La fijación óptima es un tema de debate con la mayoría de los cirujanos a favor de la fijación cementada. Aunque todavía se necesitan estudios de alta calidad a largo plazo, los avances mate-

\section{ABSTRACT}

Interest for uncemented total knee arthroplasty (TKA) has greatly increased in recent years. As the number of younger and more active patients treated with TKA continues to increase, consideration of better fixation as a means of improving implant longevity is required. This technique, less used than cemented knee replacement in the last decades, sees a revival thanks an advance in prosthetic design, instrumentation and operative technique. The related literature in some cases shows conflicting data on survival and on the revision's rate, but in most cases a success rate comparable to cemented TKA is reported. Noncemented components offer the potential advantage of a biologic interface between the bone and implants, which could demonstrate the greatest advantage in long-term durable fixation in the follow-up of young patients undergoing arthroplasty. The optimal fixation in TKA is a subject of debate with the majority of surgeons favouring cemented fixation. Although longterm, high-quality studies are still needed, material advances in biologic fixation surfaces, such as trabecular metal and hydroxyapatite, may offer promising results 
riales en las superficies de fijación biológica, como el metal trabecular y la hidroxiapatita, pueden ofrecer resultados prometedores para pacientes jóvenes y activos sometidos a artroplastia total de rodilla en comparación con las opciones cementadas tradicionales. Realizamos una revisión de la literatura que evalúa los avances de ATR no cementada y los resultados reportados.

Palabras clave: Artroplastia total de rodilla (ATR), artroplastia total de rodilla no cementada, reemplazo total de rodilla cementada. for young and active patients undergoing total knee arthroplasty when compared with traditional cemented options. We undertook a review of the literature examining advances of cementless TKA and the reported result.

Keywords: Total knee arthroplasty, noncemented total knee arthroplasty, cemented total knee arthroplasty.

\section{INTRODUCCIÓN}

La artroplastia total de rodilla (ATR) se ha convertido en uno de los procedimientos más efectivos, confiables y predecibles en la cirugía ortopédica moderna. Este procedimiento se asocia con baja morbimortalidad, y su efectividad está bien establecida para reducir el dolor articular y mejorar el rango de movimiento. Los objetivos principales de la ATR son obtener un implante que alivie el dolor, recupere la función y que satisfaga las necesidades de los pacientes. El éxito del procedimiento está bien documentado junto con sus altas tasas de satisfacción del paciente y la supervivencia del implante (entre 10 y 15 años) en más de $90 \%$. Sin embargo, algunos detalles como el abordaje quirúrgico, la técnica de fijación del dispositivo, la preservación o resección de ligamentos y los biomateriales óptimos para la fabricación de componentes individuales siguen siendo controvertidos, dentro de éstos, el uso de prótesis cementadas versus no cementadas. ${ }^{1}$

La fijación sin cemento se consideró por primera vez a mediados de la década de 1980 cuando se descubrió que el reemplazo total de rodilla no cementada en pacientes jóvenes tenía resultados intermedios comparables con la ATR cementada. La fijación sin cemento tuvo como objetivo disminuir el riesgo de aflojamiento del implante al confiar en una interfaz biológica huesoimplante. En pacientes más jóvenes (generalmente menores de 65 años) con un stock óseo adecuado, la ATR no cementada ofreció una forma de evitar las alteraciones de la interfaz hueso/cemento que tiene el potencial de provocar osteólisis. Si bien algunos resultados iniciales parecían prometedores, la fijación sin cemento pronto se relacionó con aflojamiento y falla de ATR, atribuidos a un diseño de ajuste a presión (press-fit) fallido. ${ }^{2} \mathrm{El}$ fracaso de las primeras generaciones de reemplazo de rodilla no cementada llevó a los cirujanos ortopédicos a abandonar esta técnica durante muchos años y hoy en día, la tasa porcentual de uso preferido por los cirujanos en la fijación cementada y no cementada con base en los datos de registro está muy a favor de la primera en los Estados Unidos. ${ }^{3}$

Actualmente el método de fijación ideal de la ATR todavía está en debate. La pregunta principal es si el uso de cemento es más eficiente que la fijación a 
presión en términos de garantizar una estabilidad duradera. El uso de cemento en la ATR se ha asociado con excelentes resultados clínicos y bajas tasas de aflojamiento aséptico en el seguimiento a largo plazo, y es el tipo de fijación más utilizado en el reemplazo de rodilla. Sin embargo, la falla de la interfaz cemento-hueso con la falta de capacidad de remodelación, así como el desgaste del tercer cuerpo, aumentan la preocupación sobre la durabilidad a largo plazo de la fijación cementada, particularmente en pacientes cada vez más jóvenes y activos. ${ }^{2}$ La demanda y el estrés son mayores en los pacientes jóvenes debido a la mayor esperanza y calidad de la vida. La falla en ATR se debe por lo regular a la falta de fijación del implante del lado tibial y la infección periprotésica. El aflojamiento aséptico es una complicación importante después de la ATR que puede causar altas tasas de revisión. ${ }^{3,4}$

Si bien la fijación cemento-hueso se atenúa con el tiempo, los implantes sin cemento pueden proporcionar mayor longevidad a través de la osteointegración y la retención de la capacidad de remodelación ósea, lo que potencialmente ofrece mejor supervivencia a largo plazo. La fijación sin cemento se ha preferido para pacientes más jóvenes, y aunque es más costosa, ofrece las ventajas de preservar el stock óseo y la densidad mineral a través de menor protección contra el estrés junto con una cirugía posiblemente breve y con menor uso de torniquete, así como la reducción del desgaste potencial del tercer cuerpo., ${ }^{1,2}$

La opción de utilizar una fijación no cementada ha tomado auge en los últimos años debido a múltiples razones. Se han visto buenos resultados en la fijación no cementada en la artroplastia total de cadera (ATC), lo cual ha llevado a rediseñar los implantes no cementados de la ATR con los conocimientos adquiridos en los diseños no cementados de la ATC. ${ }^{5,6}$ El objetivo de este artículo es revisar el estado actual de la literatura y los resultados reportados de la ATR no cementada.

\section{INDICACIONESY CARACTERÍSTICAS DEL IMPLANTE}

Una ATR no cementada se indica principalmente en pacientes con una buena calidad ósea y alta actividad metabólica para promover una adecuada fijación biológica. De hecho, las indicaciones más precisas son pacientes jóvenes (menores de 65 años) y con una reserva ósea adecuada.

Para garantizar una buena estabilidad primaria del implante, las resecciones óseas deben realizarse con precisión, evitando cualquier espacio entre el hueso huésped y los componentes. Por el contrario, en la ATR cementada el manto de cemento puede llenar fácilmente pequeños defectos en las resecciones sin afectar la estabilidad..$^{1,7}$

Los análisis radioestereométricos (RSA) han permitido comprender los distintos patrones de migración mostrados por los componentes de la ATR en los dos métodos de fijación diferentes. Las bases tibiales no cementadas pueden migrar temprano, es decir, en los primeros tres meses después de la operación, alcanzando la estabilidad por lo general después de este intervalo; los componentes tibiales cementados, por otro lado, no migran en el postoperatorio inme- 
diato, aunque pueden mostrar micromovimiento durante 60 meses. No se han demostrado diferencias en el patrón de migración de cementado con respecto a los componentes femorales no cementados.

Los implantes no cementados pueden llegar a ser hasta tres veces más costosos que los cementados debido a la alta tecnología requerida para producir superficies bioactivas, aunque los precios varían de una compañía a otra. Los partidarios de la ATR cementada sostienen que no es razonable usar un sistema sin cemento costoso que ofrezca los mismos resultados clínicos generales que un implante más barato, incluso en pacientes más jóvenes. Sin embargo, el uso de la ATR no cementada es sin duda un ahorro de tiempo quirúrgico, con periodo menor de isquemia neumática y por último, permite una cirugía de revisión más fácil en caso de falla. El tiempo quirúrgico más corto es un factor importante a considerar, de hecho reduce los costos asociados con la intervención. ${ }^{1,8}$

El uso de la fijación complementaria es controvertido en la ATR no cementada (Figura 1). El crecimiento óseo puede ocurrir a una distancia máxima de 0.3 a $0.5 \mathrm{~mm}$ entre el hueso y el implante. El concepto de usar tornillos para proporcionar una estabilidad inicial más predecible llevó a muchos diseños iniciales a incorporar tornillos en las bases tibiales. Sin embargo, algunos estudios revelan osteólisis asociada con el uso de tornillos y discuten los riesgos teóricos de la formación de detrito de partículas metálicas, túneles osteolíticos y daño neurovascular. Muchos cirujanos articulares ahora reconocen que los vástagos, quillas y clavijas proporcionan una fijación inicial suficiente, y el uso de platillos tibiales con tornillos es menos popular.

Las prótesis de nueva generación dan como resultado una mejor osteointegración que en el pasado, con la creación de una interfaz biológica entre el hueso y el componente protésico que puede proporcionar mejores resultados a largo plazo. De hecho, cuando se utiliza la opción sin cemento, debe evaluarse la capacidad del hueso de resistir la transmisión directa de la carga de prótesis
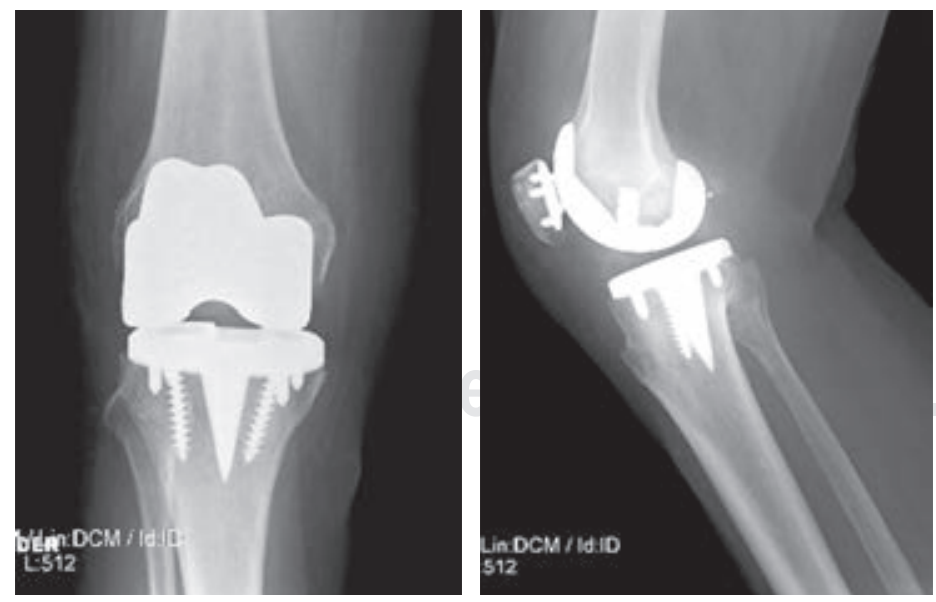

Figura 1:

Imagen radiográfica anteroposterior y lateral de ATR no cementada con fijación complementaria con tornillos. 
ósea y para estabilizar especialmente el componente tibial. Por esta razón, esta opción es preferible en pacientes jóvenes que tienen un buen stock óseo y una capacidad rápida de cicatrización ósea. Se ha demostrado que el uso de nuevas tecnologías con capas de hidroxiapatita o metal trabecular y altamente poroso puede reducir los micromovimientos y mejorar la integración entre el hueso y el componente protésico, ofreciendo mayor confiabilidad. ${ }^{1,9}$

En cuanto a la rótula con ATR, una fijación sin cemento es una práctica que rara vez se utiliza, la prótesis patentada de polietileno cementado ha mostrado excelentes resultados a largo plazo y a menudo se asocia a ATR con componentes femorales y tibiales no cementados. ${ }^{1}$

\section{ESTADO ACTUAL DE LA LITERATURA}

Actualmente, la literatura ortopédica incluye diversos estudios de casos y controles así como ensayos clínicos aleatorizados y revisiones sistemáticas que comparan la fijación sin cemento y cementada en diferentes intervalos de seguimiento.

Miller AJ y colaboradores realizaron un estudio retrospectivo de casos y controles de 400 ATR primarias en comparación con ATR no cementadas (200 pacientes) versus cementadas (200 pacientes) usando el mismo diseño de implante. El seguimiento promedio en el grupo sin cemento fue de 2.4 años (rango de dos a 3.5 años) y el grupo cementado de 5.3 años (rango de dos a 10.9 años) en quienes se evaluaron los resultados clínicos Knee Society Score (KSS) y radiográficos. Los pacientes en ambos grupos tuvieron una incidencia similar de complicaciones postoperatorias $(p=0.90)$. El grupo sin cemento tuvo siete revisiones y sólo una de ellas presentó aflojamiento aséptico del componente tibial $(0.5 \%)$. Los componentes tibiales sin cemento demostraron áreas de mayor densidad ósea en las clavijas del componente tibial. El grupo cementado tuvo en total ocho revisiones con cinco casos de aflojamiento aséptico (2.5\%). ${ }^{10}$

Lawrie CM y su equipo compararon el costo real entre un procedimiento de ATR cementada y no cementada en los Estados Unidos. El costo del tiempo operatorio, los implantes, el cemento y los accesorios de cementación se incluyeron dentro del costo total del procedimiento y fueron considerados a partir de datos institucionales y comerciales. El tiempo operatorio promedio para una ATR cementada fue 11.6 minutos más largo que en la ATR sin cemento (93.7 minutos (desviación estándar (DE) 16.7) vs. 82.1 minutos (DE 16.6); $p=0.001$ ). Utilizaron un estándar de costo por minuto del tiempo de quirófano que fue de \$36 USD; el costo total del tiempo fue \$418 USD más alto para una ATR cementada. El costo del cemento y los accesorios osciló entre \$170 y \$625 USD. En general, el costo calculado de la ATR cementada fue de $\$ 588$ a $\$ 1,043$ USD, según la técnica. El costo general aumentado de los implantes de una ATR no cementada sobre los implantes cementados fue de \$366 USD. La conclusión fue que el costo de procedimiento general de implantar una ATR no cementada es menor que implantar una ATR cementada. ${ }^{11}$

Nam D. y colegas compararon los resultados clínicos en un estudio prospectivo, aleatorizado y controlado en pacientes de 18 a 75 años de edad en quienes 
se utilizaron las versiones cementadas y no cementadas de un mismo diseño de ATR. Se analizó un total de 141 pacientes en un periodo promedio de dos años después de la cirugía. En el transoperatorio, el tiempo quirúrgico total disminuyó en la cohorte no cementada $(82.1 \pm 16.6$ en comparación con $93.7 \pm 16.7 \mathrm{mi}-$ nutos, $p=0.001$ ), siendo estadísticamente significativo. No hubo diferencias en los resultados clínicos (escala de rodilla de Oxford, escala KSS y escala visual análoga (EVA) y a las cuatro o seis semanas, un año, o un promedio de dos años después de la operación ( $p=0.1$ a 0.9 ) entre las cohortes cementadas y no cementadas. Tampoco hay evidencia radiográfica de hundimiento o aflojamiento de componentes en ninguna de las cohortes. Este estudio demostró que una ATR no cementada recientemente introducida tuvo resultados equivalentes a los de su predecesora cementada, sin fallas asépticas de ninguno de los implantes, tanto perioperatorios como al final del seguimiento estipulado. ${ }^{12}$

Prudhon JL y colaboradores en un estudio retrospectivo de casos y controles compararon la supervivencia de una serie de 100 ATR cementadas con una serie similar de 100 de no cementadas (recubrimiento de hidroxiapatita) con un seguimiento de 11-16 años, todas con diseño de platillo móvil y posteroestabilizadas. El análisis de supervivencia se determinó mediante la prueba de log-rank. El análisis de supervivencia a los 11 años de seguimiento mostró: grupo cementado: 90.2\% IC95\% [81.9-94.8]; grupo no cementado: 95.4\% IC95\% [88.1-98.2]. En la comparación entre ambos grupos no hubo una diferencia estadísticamente significativa, $\mathrm{p}=0.32 .{ }^{13}$

Debido a que la fijación ideal para los componentes tibiales modernos en la ATR sigue siendo controvertida, Bahery OA y su equipo realizaron un estudio retrospectivo de casos y controles en el que se compararon 70 casos con ATR no cementada con 70 casos de ATR cementada de 2008 a 2015 en función del diseño del implante y la demografía del paciente. La ATR no cementada mostró mayor aflojamiento aséptico (siete frente a $0, p=0.013$ ) y cirugía de revisión (10 frente a $0, p=0.001$ ) que la fijación cementada posterior a un seguimiento de cinco años, pero sin diferencias clínicamente significativas en el resultado funcional (KSS). ${ }^{2}$

Harwin SF y colegas evaluaron de manera prospectiva la supervivencia del implante y los resultados de una serie de 104 pacientes con 107 casos consecutivos con ATR no cementada de hidroxiapatita posteroestabilizada realizada por un solo cirujano entre 2008 y 2010, con un seguimiento mínimo de siete años (de siete a nueve años). La supervivencia fue de 98\% (IC 95\%: 1.01-0.96). Se reportó un aflojamiento postraumático de la placa base tibial y una revisión por inestabilidad. No hubo revisiones femorales o de la patela. La puntuación media de dolor con la escala de la Knee Society fue de 93 puntos (rango, 80-100 puntos) y la puntuación de la función fue de 78 puntos (KSS) (rango, 68-95 puntos). Excluyendo las revisiones, no hubo evidencia de aflojamiento progresivo de ningún componente del implante. ${ }^{9}$

Fricka KB y colaboradores realizaron un ensayo clínico controlado aleatorizado no cegado en el que se incluyó un total 100 pacientes, se comparó la ATR cementada y no cementada. ${ }^{14}$ Las escalas KSS, Oxford y la EVA se recolectaron 
antes y después de la operación. Se obtuvo un seguimiento de dos años para 93 pacientes. La EVA media mostró una tendencia más alta para el grupo sin cemento a los cuatro meses $(p=0.06)$. A los dos años, los puntajes funcionales de KSS, los puntajes de Oxford y las preguntas autoinformadas de satisfacción, menos dolor y mejor función fueron similares, pero el grupo cementado tuvo puntajes clínicos de KSS más altos (96.4 vs. 92.3, $p=0.03$ ). Se observaron más radiolucidencias en las rodillas no cementadas $(p<0.001)$. El grupo no cementado tuvo una revisión de inestabilidad y una rodilla cementada fue revisada por infección. La ATR no cementada mostró una supervivencia equivalente en comparación con la ATR cementada en este seguimiento temprano. ${ }^{6}$

Chen C y su equipo realizaron en 2018 un metaanálisis en ensayos clínicos controlados (ECC) cuyo objetivo fue comparar la fijación en ATR no cementada y cementada en pacientes jóvenes. Las medidas de resultado consistieron en resultados funcionales, Knee Society Score, rango de movimiento, resultados radiológicos, evaluación del dolor y complicaciones. Cuatro ECC cumplieron nuestros criterios de inclusión con 255 pacientes con ATR cementada y 229 pacientes con ATR no cementada. El metaanálisis indicó que había una diferencia significativa entre los grupos en términos de resultados radiológicos y puntaje de dolor en favor de la ATR no cementada $(p<0.03)$. No se encontraron diferencias significativas con respecto a KSS, rango de movimiento o complicaciones (aflojamiento aséptico) $(p>0.80) .^{3}$

Newman JM y colegas hicieron una revisión de la literatura en la que evaluaron los primeros y los más recientes implantes no cementados resultado de los diseños actuales, los riesgos y beneficios, y compararon los nuevos implantes no cementados versus los cementados. Para el análisis final se incluyeron 31 estudios publicados desde 2010 hasta 2017. Éstos mostraron que los pacientes con ATR cementada y no cementada tuvieron resultados similares en varios momentos postoperatorios. La satisfacción del paciente y los resultados con diversas escalas funcionales también fueron similares entre todas las cohortes. Por lo tanto, la ATR no cementada resulta ser igualmente efectiva que la ATR cementada. Los diseños más recientes de ATR no cementada han demostrado una excelente supervivencia, resultados funcionales y tasas de satisfacción en poblaciones jóvenes y de edad avanzada. En comparación con la ATR cementada, puede haber beneficios con los implantes más recientes sin cemento. Sin embargo, estos hallazgos deben justificarse aún más con estudios adicionales que reporten resultados a más largo plazo. ${ }^{15}$

\section{CONCLUSIONES}

La ATR no cementada representa una alternativa interesante, especialmente para el paciente menor de 65 años, en quien es deseable una fijación biológica prótesis-ósea, evitando los inconvenientes de la fragmentación del cemento y la posible revisión futura de un implante cementado. Estudios recientes demuestran la capacidad de la ATR no cementada de lograr excelentes resultados clínicos y de conducir a los cirujanos a optar por esta técnica de fijación con la selección 
adecuada del paciente. Si bien los dos tipos de fijación muestran resultados clínicos comparables, la fijación sin cemento ofrece, al parecer, una ventaja económica. Sin embargo, a medida que las características demográficas del paciente típico con ATR continúan cambiando, nos alientan a realizar más estudios clínicos aleatorizados de alta calidad y a más largo plazo para comparar directamente la fijación sin cemento versus la fijación cementada en una población de pacientes jóvenes. En México aún no contamos con la disponibilidad y la experiencia en el uso de implantes no cementados, por lo que estos resultados nos serán de utilidad en la toma de decisiones para ofrecer a nuestros pacientes implantes con mayor supervivencia, costo-efectividad y con menos complicaciones.

\section{BIBLIOGRAFÍA}

1. Aprato A, Risitano S, Sabatini L, Giachino M, Agati G, Massè A. Cementless total knee arthroplasty. Ann Transl Med. 2016; 4 (7): 129.

2. Behery OA, Kearns SM, Rabinowitz JM, Levine BR. Cementless vs cemented tibial fixation in primary total knee arthroplasty. J Arthroplasty 2017; 32 (5): 1510-1515.

3. Chen C, Li R. Cementless versus cemented total knee arthroplasty in young patients: a meta-analysis of randomized controlled trials. J Orthop Surg Res 2019; 14 (1): 262.

4. Fernández-Cuadrod ME. Análisis de la calidad de vida en pacientes con prótesis total de rodilla (Postgrado). Universidad de Salamanca (España); 2013. Disponible en: https://gredos.usal.es/ handle/10366/124148.

5. Dalury DF. Cementless total knee arthroplasty. Bone Joint J. 2016; 98-B: 867-873.

6. Fricka KB, Sritulanondha S, McAsey CJ. To cement or not? two-year results of a prospective, randomized study comparing cemented vs cementless total knee arthroplasty (TKA). J Arthroplasty. 2015; 30 (9 Suppl): 55-58.

7. Papas PV, Congiusta D, Cushner FD. Cementless versus cemented fixation in total knee arthroplasty. J Knee Surg. 2019; 32 (7): 596-599.

8. Ponziani L, Di Caprio F, Meringolo R. Cementless knee arthroplasty. Acta Biomed. 2017; 88 (4 Suppl): $11-18$

9. Harwin SF, Levin JM, Khlopas A, Ramkumar PN, Piuzzi NS, Roche M, et al. Cementless posteriorly stabilized total knee arthroplasty: seven-year minimum follow-up report. J Arthroplasty. 2018; 33 (5): 1399-1403.

10. Miller AJ, Stimac JD, Smith LS, Feher AW, Yakkanti MR, Malkani AL. Results of cemented vs cementless primary total knee arthroplasty using the same implant design. J Arthroplasty. 2018; 33 (4): 1089-1093.

11. Lawrie CM, Schwabe M, Pierce A, Nunley RM, Barrack RL. The Cost of implanting a cemented versus cementless total knee arthroplasty. Bone Joint J. 2019; 101-B (7 Supple C): 61-63.

12. Nam D, Lawrie CM, Salih R, Nahhas CR, Barrack RL, Nunley RM. Cemented versus cementless total knee arthroplasty of the same modern design. J Bone Joint Surg Am. 2019; 101 (13): 1185-1192.

13. Prudhon JL, Verdier R. Cemented or cementless total knee arthroplasty? Comparative results of 200 cases at a minimum follow-up of 11 years. Sicot J 2017; 3: 70.

14. Karachalios T, Antoniou I. Long term outcome of total knee arthroplasty. The effect of implant fixation (Cementless). En: Karachalios T (eds). Total knee arthroplasty. Springer, London; Imprint Springer, 2015. p. 155-162.

15. Newman JM, Sodhi N, Khlopas A, Sultan AA, Chughtai M, Abraham R, et al. Cementless total knee arthroplasty: a comprehensive review of the literature. Orthopedics. 2018; 41 (5): 263-273. 